\title{
BMJ Open Association between dementia parental family history and mid-life modifiable risk factors for dementia: a cross- sectional study using propensity score matching within the Lifelines cohort
}

\author{
Joyce Vrijsen (D) , ${ }^{1}$ Ameen Abu-Hanna, ${ }^{2}$ Sophia E de Rooij, ${ }^{3}$ Nynke Smidt ${ }^{1}$
}

To cite: Vrijsen J, Abu-Hanna A, de Rooij SE, et al. Association between dementia parental family history and mid-life modifiable risk factors for dementia: a cross-sectional study using propensity score matching within the Lifelines cohort. BMJ Open 2021;11:e049918. doi:10.1136/ bmjopen-2021-049918

- Prepublication history and additional supplemental material for this paper are available online. To view these files, please visit the journal online (http://dx.doi.org/10.1136/ bmjopen-2021-049918).

Received 06 February 2021 Accepted 19 November 2021

Check for updates

(c) Author(s) (or their employer(s)) 2021. Re-use permitted under CC BY-NC. No commercial re-use. See rights and permissions. Published by BMJ.

${ }^{1}$ Epidemiology, University of Groningen, University Medical Centre Groningen, Groningen, The Netherlands

${ }^{2}$ Medical Informatics, University of Amsterdam, Amsterdam UMC Amsterdam, The Netherlands ${ }^{3}$ Internal Medicine, University of Groningen, University Medical Centre Groningen, Groningen, The Netherlands

Correspondence to

Joyce Vrijsen; j.vrijsen@umcg.nl

\section{ABSTRACT}

Objective Individuals with a parental family history (PFH) of dementia have an increased risk to develop dementia, regardless of genetic risks. The aim of this study is to investigate the association between a PFH of dementia and currently known modifiable risk factors for dementia among middle-aged individuals using propensity score matching (PSM).

Design A cross-sectional study.

Setting and participants A subsample of Lifelines (3565 years), a prospective population-based cohort study in the Netherlands was used.

Outcome measures Fourteen modifiable risk factors for dementia and the overall Lifestyle for Brain Health (LIBRA) score, indicating someone's potential for dementia risk reduction (DRR).

Results The study population included 89869 participants of which 10940 (12.2\%) had a PFH of dementia (mean (SD) age=52.95 (7.2)) and 36389 $(40.5 \%$ ) without a PFH of dementia (mean (SD) age $=43.19$ (5.5)). Of 42540 participants (47.3\%), PFH of dementia was imputed. After PSM, potential confounding variables were balanced between individuals with and without PFH of dementia. Individuals with a PFH of dementia had more often hypertension $(\mathrm{OR}=1.19 ; 95 \% \mathrm{Cl} 1.14$ to 1.24$)$, high cholesterol $(\mathrm{OR}=1.24 ; 95 \% \mathrm{Cl} 1.18$ to 1.30$)$, diabetes (OR=1.26; $95 \% \mathrm{Cl} 1.11$ to 1.42 ), cardiovascular diseases $(\mathrm{OR}=1.49 ; 95 \% \mathrm{Cl} 1.18$ to 1.88$)$, depression $(\mathrm{OR}=1.23$; $95 \% \mathrm{Cl} 1.08$ to 1.41$)$, obesity (OR=1.14; $95 \% \mathrm{Cl} 1.08$ to 1.20 ) and overweight ( $\mathrm{OR}=1.10 ; 95 \% \mathrm{Cl} 1.05$ to 1.17$)$, and were more often current smokers $(\mathrm{OR}=1.20 ; 95 \%$ $\mathrm{Cl} 1.14$ to 1.27 ) and ex-smokers (OR=1.21; $95 \% \mathrm{Cl} 1.16$ to 1.27). However, they were less often low/moderate alcohol consumers $(\mathrm{OR}=0.87 ; 95 \% \mathrm{Cl} 0.83$ to 0.91$)$, excessive alcohol consumers ( $\mathrm{OR}=0.93 ; 95 \% \mathrm{Cl} 0.89$ to $0.98)$, socially inactive $(0 \mathrm{R}=0.84 ; 95 \% \mathrm{Cl} 0.78$ to 0.90$)$ and physically inactive (OR=0.93; $95 \% \mathrm{Cl} 0.91$ to 0.97$)$. Having a PFH of dementia resulted in a higher LIBRA score ( $\mathrm{RC}=0.15 ; 95 \% \mathrm{Cl} 0.11$ to 0.19 ).

Conclusion We found that having a PFH of dementia was associated with several modifiable risk factors. This suggests that middle-aged individuals with a PFH of dementia are a group at risk and could benefit from DRR Further research should explore their knowledge, beliefs and attitudes towards DRR, and whether they are willing
Strengths and limitations of this study

- No other study investigating the association between a parental family history of dementia and modifiable risk factors for dementia used a wide range of the currently known modifiable risk factors for dementia.

- Our large study sample provided sufficient power to detect relevant associations independent of confounding factors.

- We used sophisticated statistical techniques to prevent selection bias and calculated ORs and regression coefficients with $95 \%$ Cls.

- Parental family history of dementia was based on self-reported questionnaires, which could have led to misclassification.

- Results were based on cross-sectional data in which previous health behaviours were not taken into account.

to assess their risk and change their lifestyle to reduce dementia risk.

\section{INTRODUCTION}

Since the world's population is ageing, the total number of people with dementia will increase. ${ }^{1}$ In 2019, around 50 million people were living with dementia worldwide and the number of people with dementia is expected to increase to 152 million by $2050 .^{2}$ Since treatment options for curing dementia are unavailable to date, prevention of dementia is the key in decreasing the burden of dementia. It is estimated that delaying dementia onset by 1 year would reduce the total worldwide number of people with dementia over 60 years old in 2050 by $11.8 \%{ }^{3}$

Accumulating evidence shows that the development of dementia is a long-term pathological process that starts approximately 10-20 years before dementia is 
clinically diagnosed. ${ }^{4-6}$ The evidence of modifiable risk factors influencing this process has been mounting. ${ }^{178}$ Livingston et al found that $40 \%$ of the dementia cases are attributable to several lifestyle-related risk factors (ie, less education, hypertension, hearing impairment, smoking, obesity, depression, physical inactivity, diabetes, low social contact, excessive alcohol consumption, traumatic brain injury and air pollution). ${ }^{9}$ Also support for several other factors was found, such as hyperlipidaemia, coronary heart disease, renal dysfunction, Mediterranean diet, cognitive activity and stress. ${ }^{810}$ The majority of these risk factors were combined in the Lifestyle for Brain Health (LIBRA) score, reflecting someone's potential for dementia risk reduction (DRR)..$^{811-13}$

Several multidomain interventions to reduce dementia risk and prevent cognitive decline among older individuals were conducted; however, only small or nonsignificant effects on cognition were found. ${ }^{14-16}$ These multidomain interventions may be more effective among cognitively healthy middle-aged individuals with a higher risk for developing dementia, for instance, individuals with a parental family history (PFH) of dementia. The average lifetime risk of developing dementia is $10 \%-12 \%$ and increases to $15 \%-25 \%$ for individuals with a family history of dementia. ${ }^{17}$ This increased risk can be explained by both genetic and lifestyle factors, ${ }^{18-21}$ which are passed on from parents to offspring. ${ }^{20}{ }^{22}$ The Apolipoprotein $\mathrm{E}$ (APOE) $\varepsilon 4$ allele is one of the genes to be consistently shown to increase the risk for dementia. ${ }^{23-25}$ Individuals with a PFH of dementia are more often carrier of this allele compared with individuals without a PFH of dementia. ${ }^{21}{ }^{26-29}$ Nevertheless, several studies have shown that individuals with a PFH of dementia have an increased risk, independent of their genetic risk. ${ }^{1827} 28$

Although the role of APOE genotype on dementia risk has been well studied, the risk factor of a PFH remains rarely studied. Only a few studies investigated the association between family history of dementia and modifiable risk factors for dementia. ${ }^{28} 30$ They found that family history of dementia was associated with both higher diastolic (DBP) and systolic blood pressure (SBP) and depression, ${ }^{28} 31$ while it was not associated with body mass index (BMI), serum lipid profiles (eg, total cholesterol (TC), high-density lipoprotein (HDL), low-density lipoprotein (LDL)), alcohol consumption and smoking behaviour. ${ }^{30}$ However, previous studies did not take all currently known modifiable risk factors for dementia into account and included a relatively small sample of participants. Moreover, these findings might be a result of confounding bias. Since age is an important risk factor for dementia, individuals with a PFH of dementia are often older and could therefore have more often modifiable risk factors for dementia, such as hypertension and high cholesterol levels. ${ }^{9}$ By using covariate adjustment, there is the threat that this confounding bias is not tackled sufficiently. Propensity score matching (PSM) is a sophisticated analysis technique that can reduce this bias by assembling a matched sample of people with and without a PFH of dementia, in which confounding factors are balanced between groups. ${ }^{32}$ By matching, a greater proportion of the systematic differences in characteristics of individuals with and without a PFH is eliminated compared with the commonly used covariate adjustment. ${ }^{32}$

Finding differences in modifiable risk factors for dementia among middle-aged individuals with and without a PFH of dementia might help to identify individuals with an increased risk for dementia and subsequently offer them tailor-made interventions for DRR. Therefore, the aim of this study was to investigate the association between a PFH of dementia and modifiable risk factors for dementia among middle-aged individuals from the general population.

\section{METHOD}

\section{Study population}

The Lifelines Cohort Study is a multidisciplinary prospective population-based cohort study examining, in a unique three-generation design, the health and healthrelated behaviours of 167729 persons living in the North of the Netherlands. It employs a broad range of investigative procedures in assessing the biomedical, sociodemographic, behavioural, physical and psychological factors which contribute to the health and disease of the general population, with a special focus on multimorbidity and complex genetics. ${ }^{33} 34$ The Lifelines Cohort Study was conducted according to the guidelines of the Declaration of Helsinki. All participants provided written informed consent. For the current study, we selected participants aged 35-65 years who participated in the baseline assessment and the first follow-up questionnaire.

\section{Measurement of independent and dependent variables Independent variable}

Family history of dementia was assessed during the first follow-up questionnaire, on average 1.5 years after baseline measurement with the question: 'Does your biological father and/or mother have or had one of the following diseases?' Participants could indicate whether their father and/or mother had dementia. This variable was dichotomised (yes/no). Furthermore, participants reported whether parents were deceased and the year of birth and death of their father and/or mother if applicable. In case one of the parents was deceased and no information was given about whether at least one parent had dementia, the PFH of dementia was recoded as missing. In these cases, dementia symptoms might not have been revealed yet. Therefore, it is unclear whether they would have developed dementia if they would still be alive. We attended to this by the use of multiple imputation (see the Statistical methods section).

\section{Dependent variables}

Dependent variables are risk and protective factors for dementia and are based on data collection during physical examination (SBP, DBP, body weight and length), 
a fasting blood sample (glucose, HbA1c, TC, HDL and serum creatinine) and questionnaires, including questions on demographic characteristics, health behaviours, (parental) health and medication use. Participants brought their medication to the research site, which was subsequently reported and categorised using the Anatomical Therapeutic Chemical (ATC) codes. $^{35}$

\section{Hypertension}

Hypertension was defined as: (1) SBP $>140 \mathrm{~mm} \mathrm{Hg}$, or (2) DBP >90 mm Hg, or (3) using blood pressure-lowering medication, which was based on the following ATC codes: C02 (antihypertensives), C03 (diuretics), C07 ( $\beta$-blocking agents), C08 (calcium channel blockers) and C09 (agents acting on renin-angiotensin system). ${ }^{35}{ }^{36}$ In case the recorded SBP and DBP were missing and the participant did not use blood pressure-lowering medication, the presence of hypertension was based on the answer of the selfreported questionnaire (Do you have hypertension?).

\section{High cholesterol}

High cholesterol was defined as: (1) a ratio of TC and HDL higher than $5 \mathrm{mmol} / \mathrm{L}$, or (2) use of lipid-lowering medication (ATC code C10 (lipid-modifying agents)). ${ }^{35} 36$ If TC and HDL levels were missing and the participant did not use any lipid-lowering medication, high cholesterol was based on the answer of the self-reported questionnaire (Have you ever been diagnosed with high cholesterol?).

\section{Renal dysfunction}

Renal dysfunction is categorised into: (1) low dysfunction (estimated glomerular filtration rate (eGFR) $>90 \mathrm{~mL} /$ $\min / 1.73 \mathrm{~m}^{2}$ ), (2) moderate dysfunction (eGFR 60-89 $\mathrm{mL} / \mathrm{min} / 1.73 \mathrm{~m}^{2}$ ), and (3) high dysfunction $($ eGFR $<60$ $\left.\mathrm{mL} / \mathrm{min} / 1.73 \mathrm{~m}^{2}\right){ }^{37-39}$

\section{Obesity and overweight}

BMI was calculated using measured body weight (in kilogram) and length (in centimetre) $\left(\mathrm{BMI}=\right.$ weight $/$ length $\left.^{2}\right)$. Subsequently, the presence or absence of overweight $(\mathrm{BMI} \geq 25.0)$ and obesity (BMI $\geq 30.0)$ was determined. ${ }^{4041}$

\section{Diabetes}

Diabetes mellitus was defined as: (1) glucose (fasting capillary blood) of $7.0 \mathrm{mmol} / \mathrm{L}$ or higher, or (2) HbAlc levels higher than $53 \mathrm{mmol} / \mathrm{mol}$, or (3) using blood glucose-lowering medication (ATC code A10 (drugs used in diabetes) ). ${ }^{35} 42$ In case glucose and HbA1c levels were missing and the participant did not use any glucoselowering medication, the presence of diabetes mellitus was based on the answer of the self-reported questionnaire (Do you have diabetes mellitus?).

\section{Cardiovascular diseases}

Participants reported whether they have suffered or still suffer from one of the following cardiovascular diseases (CVDs): myocardial infarction, stroke or peripheral arterial diseases. If at least one of these CVDs was indicated with 'yes' in the self-reported questionnaire, participants were known with CVDs.

\section{Healthy diet}

A quantitative Food Frequency Questionnaire was used to assess dietary intake over the previous month. ${ }^{43} 44$ Subsequently, the Lifelines Diet Score (LLDS) was used to determine adherence to a healthy diet, which is based on the consumption of nine positive food groups (vegetables, fruit, whole-grain products, legumes and nuts, fish, oils and soft margarines, unsweetened dairy, coffee and tea) and three negative food groups (red and processed meat, butter and hard margarines and sugar-sweetened beverages). The consumption of each food group was divided into quintiles to score an individual's consumption compared with the total Lifelines population. For each food group, the quintiles ranged from 0 to 4 points, using 4 points for the highest quintile of consumption for positive food groups and the lowest quintile for the negative food groups. The total LLDS ranges from 0 to 48 , with a higher score indicating a healthier $\operatorname{diet}^{45}$

\section{Alcohol consumption}

Alcohol consumption is categorised into: (1) no alcohol consumption ( 0 alcohol unit in the past month), (2) low/ moderate alcohol consumption (average $\leq 1$ alcohol unit per day and no binge drinking), and (3) excessive alcohol consumption (average $>1$ alcohol unit per day and/or binge drinking, which is defined as more than 3 alcohol units per occasion for females and more than 4 alcohol units per occasion for males).

\section{Physical inactivity}

Physical inactivity was measured with the Short Questionnaire to Assess Health-enhancing Physical Activity. ${ }^{46}$ The results are converted to minutes/week spent in physical activity of light intensity and moderate to vigorous physical activity (MVPA) intensity, based on metabolic equivalent of tasks derived from the Ainsworth's compendium of physical activity. ${ }^{47}$ Physical inactivity is defined as less than $150 \mathrm{~min} /$ week MVPA. $^{48}$

\section{Smoking}

Smoking behaviour was assessed with the self-reported questionnaire, including the following two questions: (1) 'Do you smoke now, or have you smoked in the past month?' and (2) 'Have you ever smoked for a full year?' Subsequently, smoking behaviour was categorised into: (1) non-smoker, (2) ex-smoker, and (3) current smoker. Current smokers are defined as people who reported smoking in the past month. Ex-smokers reported smoking for at least 1 year but did not smoke in the past month.

\section{Social activity}

Social activity was measured with the following question: 'On average how many people did you have contact with in the past two weeks?' Subsequently, social activity is categorised into low (contacts $<4$ ), moderate (contacts 4-7) and high (contacts $\geq 8){ }^{49}$ 


\section{Depression}

The presence of a major depression was measured with the Mini-International Neuropsychiatric Interview. ${ }^{50}$ Major depression was defined as having at least one key symptom of depression (eg, depressed mood or loss of interest) and four additional symptoms in the past month, according to the Diagnostic and Statistical Manual of Mental Disorders, Fifth Edition. ${ }^{51}$

\section{Stress}

Chronic stress was measured by the Long-term Difficulties Inventory, ${ }^{52}$ which consists of 12 items that refer to 12 stressful life events, with regard to housing, work, social relationships, free time, finances, health, school/study and religion. Participants indicated how much stress they experienced over the past 12 months with regard to each aspect on a 3 -point scale $(0=$ not stressful; $1=$ slightly stressful; 2=very stressful). Total scores range from 0 (no stress) to 24 (very stressful).

\section{LIBRA score}

The LIBRA score reflects an individual's potential to reduce their risk on developing dementia and is based on a total of 12 protective (ie, Mediterranean diet, low/ moderate alcohol consumption, high-cognitive activity) and risk factors (ie, physical inactivity, smoking, CVDs, hypertension, high cholesterol, diabetes mellitus, obesity, renal dysfunction, depression) for dementia. ${ }^{811-13}$ Using the relative risks derived from the systematic review of Deckers et al, the LIBRA score was calculated. ${ }^{8}$ Since cognitive activities were not measured in Lifelines, LIBRA scores could range from -2.7 (low risk for dementia) to 12.7 (high risk for dementia). In table 1, the definitions and corresponding scores for each protective and risk factor for dementia are presented.

\section{Covariates}

The demographic factors such as age, sex and education were measured at baseline. Age (in years) is included as a continuous variable. Sex is included as a dichotomous variable (male/female). Education was based on the question: 'What is your highest completed level of education?' Highest level of education was categorised into: (1) elementary (no education or primary education), (2) lower secondary (lower or preparatory vocational education or lower general secondary education), (3) upper secondary (intermediate vocational education), and (4) tertiary (higher general secondary education or preuniversity secondary education, higher vocational education and university). ${ }^{54}$

\section{Statistical methods}

The baseline characteristics of the total study population were described and differences between participants with and without a PFH of dementia were calculated using standardised mean differences (SMD). Five imputed data

\begin{tabular}{|c|c|c|c|}
\hline \multicolumn{2}{|c|}{ Modifiable risk factors } & Definition & Score \\
\hline \multicolumn{4}{|c|}{ Protective factors } \\
\hline 1 & Healthy diet & LLDS $\geq 5^{\text {th }}$ quintile (score of 30 and higher) & -1.7 \\
\hline 2 & No to low/moderate alcohol consumption & $\begin{array}{l}\text { Average number of alcohol units per day } \leq 1 \text { without } \\
\text { binge drinking (ie, }>3 \text { units per day for women; }>4 \text { units } \\
\text { per day for men) }\end{array}$ & -1.0 \\
\hline
\end{tabular}

\section{Risk factors}

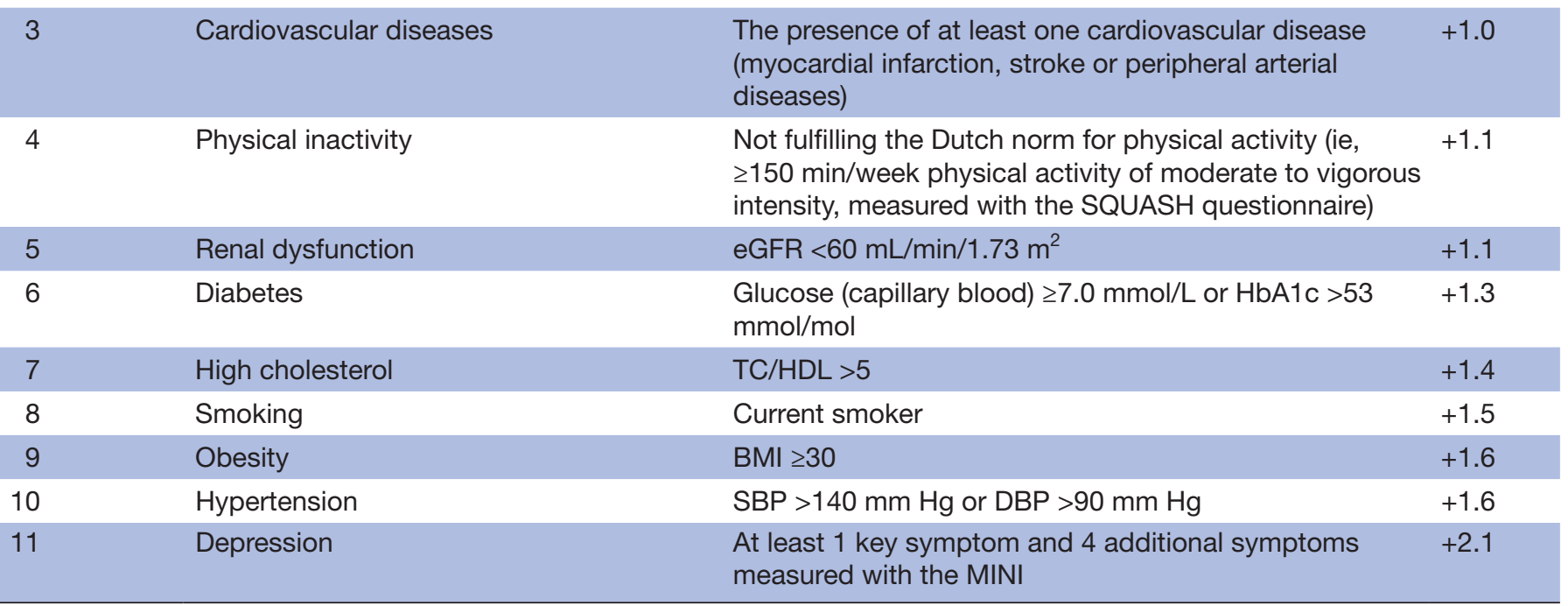

BMI, body mass index; DBP, diastolic blood pressure; eGFR, estimated glomerular filtration rate; HDL, high-density lipoprotein; LDL, lowdensity lipoprotein; LIBRA, Lifestyle for Brain Health; LLDS, Lifelines Diet Score; MINI, Mini-International Neuropsychiatric Interview; SBP, systolic blood pressure; SQUASH, Short Questionnaire to Assess Health-enhancing Physical Activity; TC, total cholesterol. 
sets were generated to replace missing values using the multiple imputation using chained equations approach. Specifically, we used predictive mean matching (ppm) for continuous data, logistic regression imputation (logreg) for binary data, polytomous regression imputation (polyreg) for unordered categorical data and proportional odds model (polr) for ordered categorical data. In each imputed data set, we assessed the association between PFH of dementia and each modifiable risk factor in two steps. First, to eliminate selection bias, PSM was used to match each individual with a PFH of dementia to an individual without a PFH of dementia (ratio 1:1) (calliper $=0.2$ ), based on the standard potential confounders age, sex and educational level (model 1) and other potential confounders (model 2) (see online supplemental file 1). ${ }^{32}$ The other potential confounders were a priori carefully selected per outcome measure in a consensus meeting, in which each potential confounder had to be associated with both the independent and the dependent variables. After PSM, we checked if the balance in the covariates was achieved (SMD <0.2). Second, logistic (dichotomous outcomes), linear (continuous outcomes) and multinomial (categorical outcomes) regression analyses were used to examine the association between a PFH of dementia and each modifiable risk factor. These analyses were conducted for each imputed matched data set to obtain the estimates, which were pooled using Rubin's rules. ${ }^{55}$ Since the LIBRA score is a composite score and includes all individual modifiable risk factors for dementia, this analysis is based on model 1 (only matched on sex, age and educational level). Results are presented as ORs or regression coefficients (RC) with 95\% CIs. Sensitivity analyses were conducted in which covariate adjustment is used instead of PSM. R statistical software environment V.1.3.383 was used ${ }^{56}$ In particular, we used the 'MatchThem', 'tableone' and 'cobalt' packages in $\mathrm{R}$.

\section{Patient and public involvement}

Participants of the Lifelines Cohort Study were not involved in the design, conduct reporting or dissemination plans of our research.

\section{RESULTS}

\section{Baseline characteristics}

A total of 106884 Lifelines participants aged 35-65 years at baseline completed the baseline assessment. For 17015 participants no data were available on PFH of dementia, since they did not participate in the first follow-up questionnaire and were therefore excluded from the analyses. This resulted in 89869 participants of which $10940(12.2 \%)$ had a PFH of dementia and $36389(40.5 \%)$ without a PFH of dementia. Of 42540 participants $(47.3 \%) \mathrm{PFH}$ of dementia was recoded as missing, since at least one parent was deceased (see the flow chart in online supplemental file 2). Table 2 presents the characteristics of participants with and without a PFH of dementia. In the observed data, we found an imbalance in age $(\mathrm{SMD}=1.534)$, education $(\mathrm{SMD}=0.271)$, hypertension $(\mathrm{SMD}=0.304)$, high cholesterol ( $\mathrm{SMD}=0.265)$, renal dysfunction $(\mathrm{SMD}=0.334)$, physical inactivity $(\mathrm{SMD}=0.375)$, diet $(\mathrm{SMD}=0.278)$ and smoking $(\mathrm{SMD}=0.333)$. After PSM on potential confounders, the balance in confounding variables was improved (see online supplemental file 3). We focused further on the results of the final model (model 2).

\section{The association between a PFH of dementia and modifiable risk factors for dementia}

The results of the logistic, linear and multinomial regression analyses on the association between a PFH of dementia and modifiable risk factors for dementia are presented in table 3. Individuals with a $\mathrm{PFH}$ of dementia had more often hypertension (OR=1.19; 95\% CI 1.14 to 1.24$)$, high cholesterol $(\mathrm{OR}=1.24 ; 95 \%$ CI 1.18 to 1.30$)$, diabetes ( $\mathrm{OR}=1.26 ; 95 \%$ CI 1.11 to 1.42$)$, CVDs ( $\mathrm{OR}=1.49 ; 95 \%$ CI 1.18 to 1.88 ), obesity ( $\mathrm{OR}=1.14 ; 95 \%$ CI 1.08 to 1.20$)$, overweight ( $\mathrm{OR}=1.10 ; 95 \%$ CI 1.05 to 1.17$)$ and depressive symptoms (OR=1.23; 95\% CI 1.08 to 1.41$)$, compared with their peers without a PFH of dementia. Further, individuals with a $\mathrm{PFH}$ of dementia were more often current smokers $(\mathrm{OR}=1.20 ; 95 \%$ CI 1.14 to 1.27$)$ and ex-smokers ( $\mathrm{OR}=1.21 ; 95 \%$ CI 1.16 to 1.27 ), but were less often low/ moderate alcohol consumers $(\mathrm{OR}=0.87 ; 95 \%$ CI 0.83 to $0.91)$, excessive alcohol consumers $(\mathrm{OR}=0.93 ; 95 \%$ CI 0.89 to 0.98$)$, physically inactive $(\mathrm{OR}=0.93 ; 95 \%$ CI 0.91 to 0.97$)$ and had less often a low social activity $(\mathrm{OR}=0.84$; $95 \%$ CI 0.78 to 0.90$)$. Finally, individuals with a PFH of dementia also had an overall higher risk to develop dementia (LIBRA score $\mathrm{RC}=0.15$; $95 \%$ CI 0.11 to 0.19 ) compared with their peers without a $\mathrm{PFH}$ of dementia.

\section{DISCUSSION}

In this study, we investigated the association between having a PFH of dementia and 14 modifiable risk factors for dementia among middle-aged individuals from the general population. We found that several modifiable risk factors for dementia were more common in individuals with a PFH of dementia independent of their age, sex and educational level. They had more often hypertension, high cholesterol, diabetes, CVDs, obesity, overweight and depression, and were also more often ex-smokers and current smokers than never smokers. However, they were more often non-alcohol consumers, physically active and socially active compared with their peers without a $\mathrm{PFH}$ of dementia. Overall, individuals with a PFH of dementia had a higher risk of developing dementia, based on the LIBRA score, which suggests that they are a group at risk for dementia.

In general, most findings are in line with our expectations, except that individuals with a PFH of dementia were less often physically and socially inactive, and less often low/moderate alcohol consumers and excessive alcohol consumers than no alcohol 
Open access

Table 2 Differences in characteristics between participants with and without a parental family history ${ }^{*}$

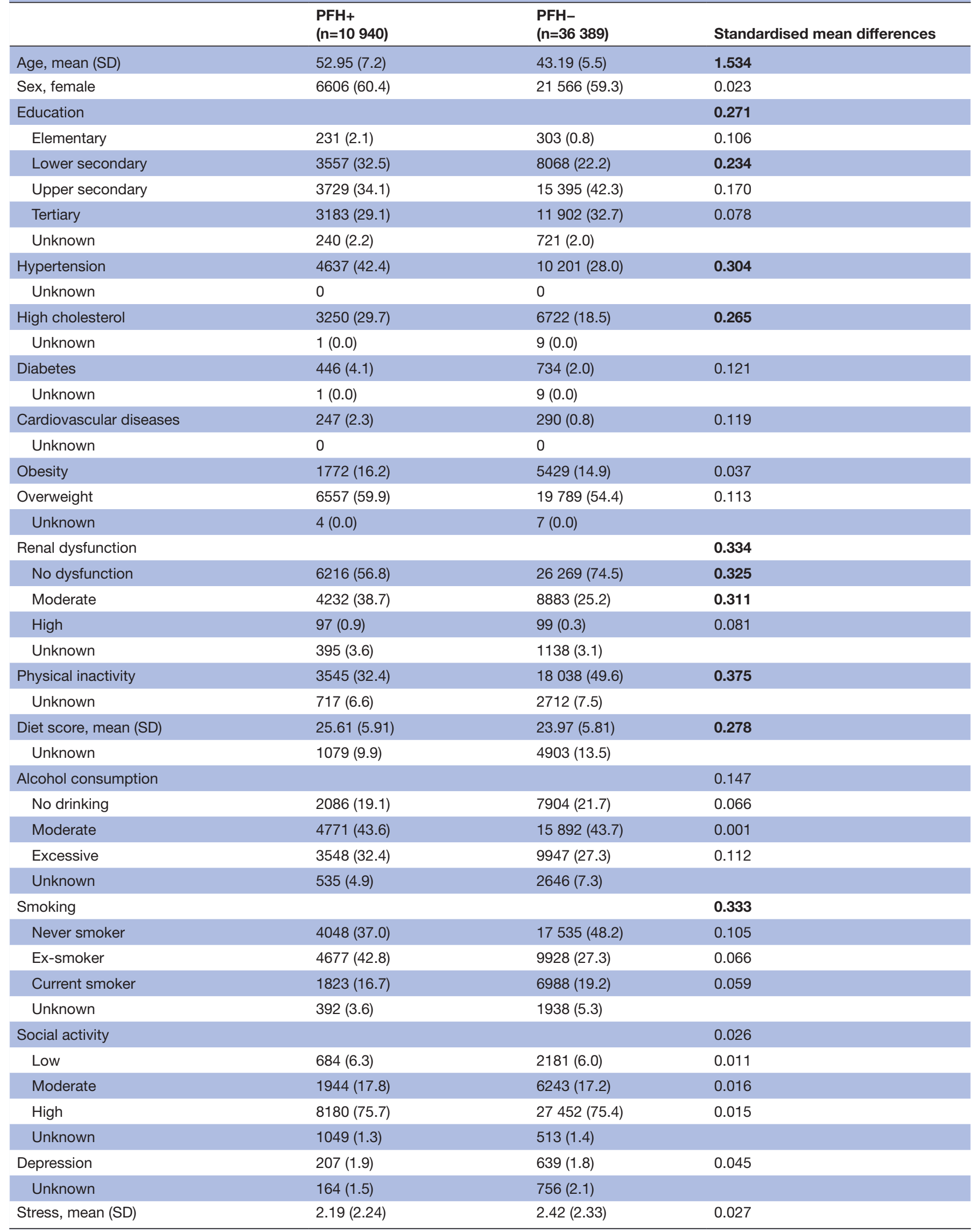

Continued 
Table 2 Continued

\begin{tabular}{llll} 
& $\begin{array}{l}\text { PFH+ } \\
(\mathbf{n}=10940)\end{array}$ & $\begin{array}{l}\text { PFH- } \\
(\mathbf{n}=\mathbf{3 6} \text { 389) }\end{array}$ & Standardised mean differences \\
\hline Unknown & $256(1.5)$ & $1066(2.0)$ & \\
\hline
\end{tabular}

Standardised mean differences higher than 0.2 are shown in bold.

${ }^{*} \mathrm{n}(\%)$ noted unless indicated otherwise.

$\mathrm{PFH}$, parental family history.

consumers. Since individuals with a PFH of dementia had more often cardiovascular risk factors, it might be that they did not consume alcohol due to health concerns or use of medication. ${ }^{57}$ Furthermore, in our study, PFH of dementia was determined by the first follow-up questionnaire. In case dementia was diagnosed before baseline assessment, individuals with a PFH of dementia could already have adjusted their lifestyle. Therefore, these findings may reflect a reverse causality from having a parent with dementia to more physical and social activity. No data were available on the date of onset of dementia.

Table 3 Results of logistic, linear and multinomial regression models assessing the association between parental family history of dementia and each modifiable risk factor for dementia

\begin{tabular}{|c|c|c|c|c|}
\hline & \multicolumn{2}{|l|}{$\begin{array}{l}\text { Without PSM } \\
\text { OR }(95 \% \mathrm{Cl})\end{array}$} & \multicolumn{2}{|l|}{$\begin{array}{l}\text { With PSM } \\
\text { OR (95\% Cl) }\end{array}$} \\
\hline & $\begin{array}{l}\text { Observed data } \\
(n=47 \text { 329) }\end{array}$ & $\begin{array}{l}\text { Imputed data } \\
(\mathrm{n}=89 \text { 869) }\end{array}$ & $\begin{array}{l}\text { Model } 1^{\star} \\
(n=53218)\end{array}$ & $\begin{array}{l}\text { Model } 2 \\
(n=53644)\end{array}$ \\
\hline Hypertension & $1.89(1.81$ to 1.97$)$ & $1.82(1.77$ to 1.88$)$ & $1.16(1.12$ to 1.21$)$ & $1.19(1.14$ to 1.24$) \dagger$ \\
\hline High cholesterol & 1.87 (1.78 to 1.96$)$ & 1.80 (1.74 to 1.86$)$ & 1.16 (1.10 to 1.22$)$ & $1.24(1.18$ to 1.30$) \dagger$ \\
\hline Diabetes mellitus & 2.06 (1.83 to 2.33$)$ & 2.07 (1.91 to 2.26$)$ & $1.20(1.07$ to 1.34$)$ & $1.26(1.11$ to 1.42$) \dagger$ \\
\hline CVD & 2.88 (2.42 to 3.41$)$ & 2.93 (2.58 to 3.33 ) & 1.40 (1.17 to 1.68$)$ & $1.49(1.18$ to 1.88$) \dagger$ \\
\hline Obesity & $1.10(1.04$ to 1.17$)$ & 1.21 (1.17 to 1.26$)$ & 1.14 (1.09 to 1.20$)$ & $1.14(1.08$ to 1.20$) \dagger$ \\
\hline Overweight & $1.26(1.20$ to 1.31$)$ & 1.31 (1.28 to 1.35$)$ & 1.07 (1.02 to 1.11$)$ & $1.10(1.05$ to 1.17$) \dagger$ \\
\hline \multicolumn{5}{|c|}{ Renal dysfunction (ref: no dysfunction) } \\
\hline Moderate & 2.01 (1.92 to 2.11$)$ & 1.79 (1.74 to 1.84$)$ & 1.02 (0.98 to 1.06$)$ & $1.02(0.97$ to 1.07$) \dagger$ \\
\hline High & 4.14 (3.13 to 5.49 ) & 4.10 (3.30 to 5.09$)$ & 1.32 (0.98 to 1.79$)$ & $1.28(0.96$ to 1.71$) \dagger$ \\
\hline Physical inactivity & $0.46(0.44$ to 0.48$)$ & $0.55(0.53$ to 0.56$)$ & $0.94(0.93$ to 1.00$)$ & $0.93(0.91$ to 0.97$) \ddagger$ \\
\hline $\operatorname{Diet}(\mathrm{RC} ; 95 \% \mathrm{Cl})$ & $1.63(1.50$ to 1.76$)$ & $1.13(1.05$ to 1.22$)$ & $0.27(0.11$ to 0.43$)$ & $-0.04(-0.16$ to 0.09$) \ddagger$ \\
\hline \multicolumn{5}{|l|}{ Alcohol (ref: no consumption) } \\
\hline Low/moderate & 1.14 (1.08 to 1.21$)$ & 1.02 (0.99 to 1.06$)$ & $0.87(0.82$ to 0.92$)$ & $0.87(0.83$ to 0.91$) \ddagger$ \\
\hline Excessive & 1.35 (1.27 to 1.44$)$ & 1.18 (1.14 to 1.23$)$ & $0.90(0.84$ to 0.97$)$ & $0.93(0.89$ to 0.98$) \ddagger$ \\
\hline \multicolumn{5}{|l|}{ Smoking (ref: never smoker) } \\
\hline Ex-smoker & 2.04 (1.94 to 2.14 ) & 1.83 (1.77 to 1.89$)$ & 1.19 (1.14 to 1.24$)$ & $1.21(1.16$ to 1.27$) \ddagger$ \\
\hline Current smoker & $1.13(1.06$ to 1.20$)$ & $1.22(1.18$ to 1.27$)$ & $1.16(1.11$ to 1.22$)$ & $1.20(1.14$ to 1.27$) \ddagger$ \\
\hline \multicolumn{5}{|c|}{ Social activity (ref: high activity) } \\
\hline Moderate & 1.05 (0.99 to 1.11$)$ & 0.89 (0.84 to 0.95$)$ & $0.97(0.47$ to 0.90$)$ & 0.95 (0.87 to 1.02$) \ddagger$ \\
\hline Low & 1.05 (0.96 to 1.15$)$ & $0.83(0.78$ to 0.87$)$ & $0.88(0.82$ to 0.95$)$ & $0.84(0.78$ to 0.90$) \ddagger$ \\
\hline Depression & 1.07 (0.92 to 1.26$)$ & $1.18(1.07$ to 1.30$)$ & 1.24 (1.10 to 1.40$)$ & $1.23(1.08$ to 1.41$) \S$ \\
\hline Stress (RC; 95\% Cl) & $-0.41(-0.46$ to -0.36$)$ & $-0.42(-0.45$ to -0.39$)$ & $0.03(-0.02$ to 0.07$)$ & $0.03(-0.13$ to 0.19$) \S$ \\
\hline LIBRA score (RC; 95\% Cl) & n.a. & $0.49(0.47$ to 0.51$)$ & $0.15(0.11$ to 0.19$)$ & n.a. \\
\hline
\end{tabular}

ORs with $95 \% \mathrm{Cls}$ are reported, unless stated otherwise; significant associations are shown in bold.

*Matched on age, sex and education level.

†Additionally matched on physical inactivity, diet, alcohol consumption, smoking, stress and depression.

¥Additionally matched on stress, social activity, cardiovascular diseases, diabetes, high cholesterol, hypertension and renal dysfunction.

$\S$ Additionally matched on physical inactivity, diet, stress and social activity.

CVD, cardiovascular disease; LIBRA, Lifestyle for Brain Health; n.a., not available; PSM, propensity score matching; RC, regression coefficient. 
To our knowledge, this is the first study that investigated the association between having a PFH of dementia and currently known modifiable risk factors for dementia among middle-aged individuals using a large sample size and PSM. Only few studies have been conducted to test the differences in several modifiable risk factors of dementia between individuals with and without a family history of dementia. ${ }^{28} 3031$ However, it is likely that these studies were hampered by small sample sizes of the study population. For instance, Lückhoff et al did not find differences in BMI (objectively measured), TC, HDL, LDL, alcohol intake and smoking behaviour between middle-aged individuals with $(\mathrm{n}=75)$ and without $(\mathrm{n}=505)$ a selfreported family history of dementia $(\mathrm{p}>0.05) .{ }^{30}$ van Exel et al found that middle-aged individuals with an objectively measured PFH of dementia $(n=206)$ had more often hypertension and caregiver burden stress compared with their peers $(n=200)(p<0.05){ }^{28}$ However, no differences were found in high cholesterol, glucose levels and lifestyle-related risk factors such as smoking and physical activity $(\mathrm{p}>0.05) .{ }^{28} \mathrm{La}$ Rue et al also showed that individuals with a PFH of dementia $(n=623)$ had higher cholesterol levels, higher DBP and SBP and higher depression rates compared with individuals without a PFH of dementia $(\mathrm{n}=157) \quad(\mathrm{p}<0.01){ }^{31} \quad$ Although differences with the current study could be explained by the use of different statistical methods, sensitivity analyses in which covariate adjustment is used showed similar results when using PSM (see online supplemental file 4). In comparison to the main analyses, the estimates for physical inactivity and social activity are slightly smaller in the sensitivity results. This could be explained by the smaller sample size in the main results ( $\mathrm{n}=53644$ vs $\mathrm{n}=89869$ ). Due to one-to-one matching, a relatively high number of healthy living individuals with a PFH of dementia could not be matched and therefore not included in the main analyses. A major advantage of PSM is that the balance in potential confounders can be inspected between individuals with and without a PFH of dementia before conducting the analyses. After PSM, most potential confounders were balanced between participants with and without a PFH of dementia (SMD $<0.2)$, except for the variable renal dysfunction $(\mathrm{SMD}=-0.207)$. Therefore, it is possible that the associations between having a PFH of dementia and lifestyle-related risk factors for dementia are slightly biased.

\section{Strengths and limitations}

Our large study sample provided sufficient power to detect relevant associations independent of confounding factors. In addition, no other study investigating the association between a PFH of dementia and modifiable risk factors for dementia used a wide range of the currently known modifiable risk factors for dementia. A large part of these modifiable risk factors (eg, hypertension, high cholesterol, diabetes mellitus, obesity, overweight, renal dysfunction) were objectively measured through physical examination and fasting blood samples. Further, we used sophisticated statistical techniques to prevent selection bias. The potential confounders used in PSM were carefully chosen per outcome measure. Finally, in contrast to previous studies, we reported adjusted ORs and RCs with 95\% CIs instead of $p$ values, which gives more information on the magnitude and direction of the association studied.

This study also had certain limitations. One drawback is that PFH of dementia was based on self-reported questionnaires and could have led to misclassification. Nonetheless, it is likely that the misclassification was non-differential and would have led to an underestimation of our results. Second, no data were available on the APOE genotype, which may be an important effect modifier. ${ }^{19}$ Previous literature showed that a healthy lifestyle might especially be beneficial for the cognition of APOE e4 carriers. ${ }^{1958}$ Since individuals with a PFH of dementia are more often carriers of the APOE e4 allele, a healthy lifestyle might also be especially beneficial for individuals with a PFH of dementia. Therefore, absence of APOE genotype data could have led to an underestimation of the results for APOE e4 carriers with a PFH of dementia. Third, the results were based on crosssectional data in which previous health behaviours were not taken into account. It might be possible that individuals with a PFH of dementia adopted a healthier lifestyle after their parent got diagnosed with dementia. In other words, our findings may reflect a reverse causality from $\mathrm{PFH}$ of dementia to health behaviour, indicating that our estimates may be underestimated. Finally, we imputed PFH of dementia of all participants without a PFH of dementia with at least one deceased parent. We did not distinguish in the age of death of deceased parents, since the incidence of dementia increases with age and the average age of onset of dementia differs between types of dementia. ${ }^{59}$ However, relatively young parents are less likely to develop dementia compared with older parents. Nevertheless, sensitivity analyses in which individuals with deceased fathers who survived to at least the age of 70 or mothers who survived to at least the age of 75 were assigned to the group without having a PFH of dementia instead of PFH being imputed showed similar results. ${ }^{31}$ Also, we did not take into account the age of onset of dementia of the parent(s), since the average age of onset of dementia differs between types of dementia. ${ }^{59}$ However, this might be an important effect modifier as early-onset dementia may have a stronger genetic basis. Therefore, these results could be an underestimation of the results for individuals with a parent diagnosed at an older age. Nevertheless, after excluding individuals with a parent diagnosed before the age of 70 years, the results were similar.

These findings support a high-risk prevention strategy for dementia by identifying the individuals with a PFH of dementia, screening them for modifiable risk factors for 
dementia and implementing multidomain interventions targeting these modifiable risk factors. Future studies should first explore the knowledge, beliefs and attitudes towards dementia (risk reduction) among middle-aged individuals with a PFH of dementia, and whether they are willing to assess their protective and risk factors for dementia and adopt a healthier lifestyle. Next, the effectiveness of these multidomain interventions in changing health behaviour for DRR among middle-aged individuals with a PFH of dementia should be investigated.

\section{CONCLUSION}

We found that a PFH of dementia was associated with several modifiable risk factors for dementia independent of age, sex and educational level, including hypertension, high cholesterol, diabetes mellitus, GVDs, obesity, overweight and depression. This suggests that middleaged individuals with a PFH of dementia are a group at risk for dementia and might benefit from DRR. Further research should examine knowledge, beliefs and attitudes towards DRR among middle-aged individuals with a PFH of dementia, and their willingness to address and tackle their personal risk factors for dementia in order to prevent or postpone dementia.

Acknowledgements The Lifelines Biobank initiative has been made possible by subsidy from the Dutch Ministry of Health, Welfare and Sport, the Dutch Ministry of Economic Affairs, the University Medical Centre Groningen (UMCG, The Netherlands), the University of Groningen and the Northern Provinces of the Netherlands.

Contributors JV, AA-H, SEdR and NS were involved in the design of the study. $\mathrm{JV}$ conducted the analysis with support from AA-H. JV wrote the manuscript and is guarantor. AA-H, SEdR and NS revised the manuscript. All authors read and approved the final manuscript.

Funding The authors have not declared a specific grant for this research from any funding agency in the public, commercial or not-for-profit sectors.

Competing interests None declared.

Patient consent for publication Not required.

Ethics approval The Lifelines Cohort Study was approved by the Medical Ethical Commission (METC) of the University Medical Centre Groningen (Reference number: METC2007/152). All subjects signed written informed consent and all methods were carried out in accordance with relevant guidelines and regulations for human subjects.

Provenance and peer review Not commissioned; externally peer reviewed.

Data availability statement Data are available upon reasonable request. Data may be obtained from a third party and are not publicly available. Lifelines is a facility that is open for all researchers (www.lifelines.net).

Supplemental material This content has been supplied by the author(s). It has not been vetted by BMJ Publishing Group Limited (BMJ) and may not have been peer-reviewed. Any opinions or recommendations discussed are solely those of the author(s) and are not endorsed by BMJ. BMJ disclaims all liability and responsibility arising from any reliance placed on the content. Where the content includes any translated material, BMJ does not warrant the accuracy and reliability of the translations (including but not limited to local regulations, clinical guidelines, terminology, drug names and drug dosages), and is not responsible for any error and/or omissions arising from translation and adaptation or otherwise.

Open access This is an open access article distributed in accordance with the Creative Commons Attribution Non Commercial (CC BY-NC 4.0) license, which permits others to distribute, remix, adapt, build upon this work non-commercially, and license their derivative works on different terms, provided the original work is properly cited, appropriate credit is given, any changes made indicated, and the use is non-commercial. See: http://creativecommons.org/licenses/by-nc/4.0/.

ORCID iD

Joyce Vrijsen http://orcid.org/0000-0003-1506-2266

\section{REFERENCES}

1 Livingston G, Sommerlad A, Orgeta V. The Lancet commissions dementia prevention, intervention, and care. Lancet 2017;390:2673-734.

2 World Health Organization. Dementia [Internet], 2019. Available: https://www.who.int/news-room/fact-sheets/detail/dementia

3 Brookmeyer R, Johnson E, Ziegler-Graham K, et al. Forecasting the global burden of Alzheimer's disease. Alzheimer's Dement 2007;3:186-91.

4 Singh-Manoux A, Kivimaki M, Glymour MM, et al. Timing of onset of cognitive decline: results from Whitehall II prospective cohort study. BMJ 2012;344:d7622

5 Sperling RA, Aisen PS, Beckett LA, et al. Toward defining the preclinical stages of Alzheimer's disease: recommendations from the National Institute on Aging-Alzheimer's association workgroups on diagnostic guidelines for Alzheimer's disease. Alzheimers Dement 2011;7:280-92.

6 Rajan KB, Wilson RS, Weuve J, et al. Cognitive impairment 18 years before clinical diagnosis of Alzheimer disease dementia. Neurology 2015;85:898-904.

7 Norton S, Matthews FE, Barnes DE, et al. Potential for primary prevention of Alzheimer's disease: an analysis of population-based data. Lancet Neurol 2014;13:788-94.

8 Deckers K, van Boxtel MPJ, Schiepers OJG, et al. Target risk factors for dementia prevention: a systematic review and Delphi consensus study on the evidence from observational studies. Int J Geriatr Psychiatry 2015;30:234-46.

9 Livingston G, Huntley J, Sommerlad A, et al. Dementia prevention, intervention, and care: 2020 report of the Lancet Commission. Lancet 2020;396:413-46.

10 Li X-Y, Zhang M, Xu W, et al. Midlife modifiable risk factors for dementia: a systematic review and meta-analysis of 34 prospective cohort studies. Curr Alzheimer Res 2019;16:1254-68.

11 Schiepers OJG, Köhler S, Deckers K, et al. Lifestyle for brain health (LIBRA): a new model for dementia prevention. Int $J$ Geriatr Psychiatry 2018;33:167-75.

12 Vos SJB, van Boxtel MPJ, Schiepers OJG, et al. Modifiable Risk Factors for Prevention of Dementia in Midlife, Late Life and the Oldest-Old: Validation of the LIBRA Index. J Alzheimers Dis 2017;58:537-47.

13 Deckers K, Köhler S, van Boxtel M, et al. Lack of associations between modifiable risk factors and dementia in the very old: findings from the Cambridge City over-75s cohort study. Aging Ment Health 2018;22:1-7.

14 Ngandu T, Lehtisalo J, Solomon A, et al. A 2 year multidomain intervention of diet, exercise, cognitive training, and vascular risk monitoring versus control to prevent cognitive decline in at-risk elderly people (finger): a randomised controlled trial. Lancet 2015;385:2255-63.

15 Moll van Charante EP, Richard E, Eurelings LS, et al. Effectiveness of a 6-year multidomain vascular care intervention to prevent dementia (preDIVA): a cluster-randomised controlled trial. Lancet 2016;388:797-805.

16 Andrieu S, Guyonnet S, Coley N, et al. Effect of long-term omega 3 polyunsaturated fatty acid supplementation with or without multidomain intervention on cognitive function in elderly adults with memory complaints (MAPT): a randomised, placebo-controlled trial. Lancet Neurol 2017;16:377-89.

17 Goldman JS, Hahn SE, Catania JW, et al. Genetic counseling and testing for Alzheimer disease: joint practice guidelines of the American College of medical genetics and the National Society of genetic Counselors. Genet Med 2011;13:597-605.

18 Lourida I, Hannon E, Littlejohns TJ, et al. Association of lifestyle and genetic risk with incidence of dementia. JAMA 2019;322:430.

19 Kivipelto M, Rovio S, Ngandu T, et al. Apolipoprotein E epsilon4 magnifies lifestyle risks for dementia: a population-based study. J Cell Mol Med 2008;12:2762-71.

20 Borenstein AR, Copenhaver CI, Mortimer JA. Early-life risk factors for Alzheimer disease. Alzheimer Dis Assoc Disord 2006;20:63-72.

21 Donix M, Small GW, Bookheimer SY. Family History and APOE-4 Genetic Risk in Alzheimer's Disease. Neuropsychol Rev 2012;22:298-309. 
22 Muñoz M, Pong-Wong R, Canela-Xandri O, et al. Evaluating the contribution of genetics and familial shared environment to common disease using the UK Biobank. Nat Genet 2016;48:980-3.

23 Corder EH, Saunders AM, Risch NJ, et al. Protective effect of apolipoprotein E type 2 allele for late onset Alzheimer disease. Nat Genet 1994;7:180-4.

24 Farrer LAet al. Effects of age, sex, and ethnicity on the association between apolipoprotein $\mathrm{E}$ genotype and Alzheimer disease. JAMA 1997;278:1349.

25 Choudhury P, Ramanan VK, Boeve BF. APOE \& 4 Allele Testing and Risk of Alzheimer Disease. JAMA 2021;325:484.

26 Sager MA, Hermann B, La Rue A. Middle-aged children of persons with Alzheimer's disease: APOE genotypes and cognitive function in the Wisconsin Registry for Alzheimer's prevention. $J$ Geriatr Psychiatry Neurol 2005;18:245-9.

27 Scarabino D, Gambina G, Broggio E, et al. Influence of family history of dementia in the development and progression of late-onset Alzheimer's disease. Am J Med Genet Part B Neuropsychiatr Genet 2016;171:250-6.

28 van Exel E, Eikelenboom P, Comijs $\mathrm{H}$, et al. Vascular factors and markers of inflammation in offspring with a parental history of lateonset Alzheimer disease. Arch Gen Psychiatry 2009;66:1263-70.

29 Johnson SC, Koscik RL, Jonaitis EM. The Wisconsin Registry for Alzheimer's Prevention: A review of findings and current directions. Alzheimer's Dement 2018;10:130-42.

30 Lückhoff HK, Kidd M, van Rensburg SJ, et al. Apolipoprotein E genotyping and questionnaire-based assessment of lifestyle risk factors in dyslipidemic patients with a family history of Alzheimer's disease: test development for clinical application. Metab Brain Dis 2016;31:213-24.

31 RueA L, Hermann B, Jones JE. Effect of parental family history of Alzheimer's disease on serial position profiles. Alzheimer's Dement 2008;4:285-90.

32 Austin PC. An introduction to propensity score methods for reducing the effects of confounding in observational studies. Multivariate Behav Res 2011;46:399-424.

33 Scholtens S, Smidt N, Swertz MA, et al. Cohort profile: lifelines, a three-generation cohort study and Biobank. Int J Epidemiol 2015;44:1172-80.

34 Stolk RP, Rosmalen JGM, Postma DS, et al. Universal risk factors for multifactorial diseases. Eur J Epidemiol 2008;23:67-74.

35 World Health Organization. ATC/DDD index. Oslo, Norway, 2020.

36 van Dis I, Kromhout D, Geleijnse JM, et al. Evaluation of cardiovascular risk predicted by different score equations: the Netherlands as an example. Eur J Cardiovasc Prev Rehabil 2010;17:244-9.

37 De Grauw W, De Leest K, Schenk P, et al. NHG-Standaard Chronische Nierschade. TPO - Prakt 2018;13:26-9.

38 Levey AS, Stevens LA, Schmid CH, et al. A new equation to estimate glomerular filtration rate. Ann Intern Med 2009;150:604-12.

39 Levey AS, Inker LA, Coresh J. Gfr estimation: from physiology to public health. Am J Kidney Dis 2014;63:820-34.

40 Centers for Disease Control and Prevention (CDC). Body Mass Index (BMI) [Internet], 2020. Available: https://www.cdc.gov/healthyweight/ assessing/bmi/adult_bmi/index.html

41 World Health Organization (WHO). Body mass index (BMI) [Internet]. World Health Organization. Available: http://www.euro.who.int/en/ health-topics/disease-prevention/nutrition/a-healthy-lifestyle/bodymass-index-bmi [Accessed 06 May 2019].

42 Rutten G, De Grauw W, Nijpels G. NHG-Standaard diabetes mellitus type 2, 2013.

43 Molag ML, de Vries JHM, Duif N, et al. Selecting informative food items for compiling food-frequency questionnaires: comparison of procedures. Br J Nutr 2010;104:446-56.

44 Siebelink E, Geelen A, de Vries JHM. Self-reported energy intake by FFQ compared with actual energy intake to maintain body weight in 516 adults. Br J Nutr 2011:106:274-81.

45 Vinke PC, Corpeleijn E, Dekker LH, et al. Development of the food-based lifelines diet score (LLDS) and its application in 129,369 lifelines participants. Eur J Clin Nutr 2018;72:1111-9.

46 Wendel-Vos GCW, Schuit AJ, Saris WHM, et al. Reproducibility and relative validity of the short questionnaire to assess health-enhancing physical activity. J Clin Epidemiol 2003;56:1163-9.

47 Ainsworth BE, Haskell WL, Herrmann SD, et al. 2011 compendium of physical activities: a second update of codes and Met values. Med Sci Sports Exerc 2011;43:1575-81.

48 Weggemans RM, Backx FJG, Borghouts L, et al. The 2017 Dutch physical activity guidelines. Int J Behav Nutr Phys Act 2018;15:58.

49 Kuiper JS, Zuidersma M, Oude Voshaar RC, et al. Social relationships and risk of dementia: a systematic review and meta-analysis of longitudinal cohort studies. Ageing Res Rev 2015;22:39-57.

50 Sheehan DV, Lecrubier Y, Sheehan KH, et al. The Mini-International neuropsychiatric interview (M.I.N.I.): the development and validation of a structured diagnostic psychiatric interview for DSM-IV and ICD10. J Clin Psychiatry 1998;59 Suppl 20:22-33.

51 American Psychiatric Association. Diagnostic and statistical manual of mental disorders (DSM-5). Fifth ed. Washington, 2013.

52 Rosmalen JGM, Bos EH, de Jonge P. Validation of the long-term difficulties inventory (LDI) and the list of threatening experiences (LTE) as measures of stress in epidemiological population-based cohort studies. Psychol Med 2012;42:2599-608.

53 Motrico E, Moreno-Küstner B, de Dios Luna J, et al. Psychometric properties of the List of Threatening Experiences--LTE and its association with psychosocial factors and mental disorders according to different scoring methods. J Affect Disord 2013;150:931-40.

54 Klijs B, Kibele EUB, Ellwardt L, et al. Neighborhood income and major depressive disorder in a large Dutch population: results from the lifelines cohort study. BMC Public Health 2016;16:773.

55 White IR, Royston P, Wood AM. Multiple imputation using chained equations: issues and guidance for practice. Stat Med 2011;30:377-99.

56 R Core team. R: a language and environment for statistical computing. Vienna, Austria, 2020.

57 Green CA, Polen MR. The health and health behaviors of people who do not drink alcohol. Am J Prev Med 2001;21:10.1016/S07493797(01)00365-8:298-305.

58 Dekhtyar S, Marseglia A, Xu W, et al. Genetic risk of dementia mitigated by cognitive reserve: a cohort study. Ann Neurol 2019;86:68-78.

59 Ott A, Breteler MM, van Harskamp F, et al. Incidence and risk of dementia. The Rotterdam study. Am J Epidemiol 1998;147:10.1093/ oxfordjournals.aje.a009489:574-80. 